\title{
Intermittent Deceleration
}

National Cancer Institute

\section{Source}

National Cancer Institute. Intermittent Deceleration. NCI Thesaurus. Code C92816.

Fetal heart rate decelerations that occur with less than 50 percent of uterine contractions within a 20 minute timeframe. 